ISSN 1997-5902

\title{
Potentiel de production fourragère d'une zone pâturée menacée de destruction : cas du cordon littoral Port- Bouët et Grand-Bassam.
}

\author{
Kouassi Akossoua Faustine ${ }^{1,3^{*}}$, Koffi Kouao Jean ${ }^{2}$, N'Goran Koua Serge Béranger 1,3, Ipou Ipou Joseph 1,3 \\ ${ }^{1}$ Centre National de Floristique, Côte d'Ivoire, 22 BP 582 Abidjan 22 \\ 2Université Nangui Abrogoua Abobo, Côte d'Ivoire, \\ 3Université Félix Houphouet Boigny, Côte d'Ivoire, 22 BP 582 Abidjan 22 \\ *Auteur correspondant e-mail : akossouafifi@yahoo.fr Tel : (+225) 47589664 \\ Original submitted in on 14th July 2014. Published online at www.m.elewa.org on 31st October 2014. \\ http://dx.doi.org/10.4314/jab.v82i1.13
}

\section{RÉSUMÉ}

Objectif : Une étude sur l'alimentation des bovins a été menée sur l'axe Port-Bouët - Grand-Bassam (Abidjan, Côte d'Ivoire), afin de montrer le potentiel de production fourragère de la zone pâturée.

Méthodologie et Résultats : L'étude a consisté à la détermination de la biomasse de la zone de pâture à travers la méthode de la récolte intégrale dans des placeaux de $1 \mathrm{~m}^{2}$ disposés au hasard dans les placettes de 625 $\mathrm{m}^{2}$ reparties sur le site d'étude. La productivité fourragère de la zone pâturée a été calculée à partir de la biomasse obtenue. Cela nous a permis de calculer la capacité de charge en UBT/ha qui correspond au nombre de têtes de bétail qu'un parcours peut accueillir et nourrir sans se détériorer. L'analyse des données obtenues a permis d'obtenir $27,05 \%$ d'espèces végétales à bonne valeur fourragère. Les espèces à valeur fourragère moyenne sont plus nombreuses avec un pourcentage de 49,42. La valeur pastorale de la zone de pâture calculée est de 40,91 avec une capacité de charge de 0,96 UBT/ha. Cela signifie qu'en moyenne un hectare de la zone d'étude peut nourrit correctement un bovin de $250 \mathrm{~kg}$ de poids vif.

Conclusions et Application des résultats : L'ensemble des espèces fourragères et la capacité de charge obtenue, confère un bon potentiel de production fourragère. Cette production est suffisante pour que la zone pâturée du cordon littoral Port-Bouët - Grand-Bassam menacée de destruction soit protégée et valorisée.

Mots clés : Production fourragère, zone pâturée, Cordon littoral, Élevage, Productivité.

(Potential forage production of a grazed area threatened with destruction: The case of barrier beach Port-Bouet and Grand-Bassam.)

\begin{abstract}
Objective: A study on the feeding of cattle was undertaken on the axis Port -Bouët - Grand- Bassam (Abidjan, Côte d' Ivoire). It aimed to show the potential of forage production of the zone for the pastoralism.

Methodology and Results: The study consisted in determining the biomass of the grazing area through the method of full harvest in sub-plots of $1 \mathrm{~m}^{2}$ spread in the plots of $625 \mathrm{~m}^{2}$ over the study area. Forage productivity of the grazed area was calculated from the biomass obtained. This allowed calculation of the carrying capacity TLU / ha corresponding to the number of cattle which can accommodate and feed without deteriorating. The
\end{abstract}




\section{Kouassi et al. J. Appl. Biosci. 2014. Potentiel de production fourragère d'une zone pâturée menacée de destruction : cas du cordon littoral Port-Bouët et Grand -Bassam}

data analysis identified that good forage plant species had a recorded value of $27.05 \%$. Species with medium feed value outnumber with a percentage of 49.42. The pastoral value of the grazing area was calculated at 40.91 with a capacity of 0.96 TLU/ha.

Conclusions and Application of Results: All forage species and the capacity obtained, gave a good potential for forage production. This capacity meant that on average one hectare of the study area can properly feed cattle of $250 \mathrm{~kg}$ live weight. This production is sufficient for the grazed area along the shoreline Port-Bouët Grand-Bassam that is threatened with destruction and should be protected and valued.

Keywords: forage production, grazing area, barrier beach, Breeding, Productivity.

\section{INTRODUCTION}

L'agriculture urbaine et périurbaine constitue un enjeu majeur en termes d'approvisionnement des villes, d'emploi et de gestion de l'environnement urbain. Elle possède des spécificités par rapport à l'agriculture rurale et apporte une contribution essentielle à l'économie domestique des villes (Moustier et Mbaye, 1999). En agriculture urbaine, l'élevage urbain constitue souvent le secteur le mieux représenté après le maraîchage (Mougeot, 2000). II forme une composante non négligeable d'un système complexe de sécurité alimentaire et financière (Waters-Bayers, 2000). En Côte d'Ivoire où, bien que l'élevage en général soit très peu développé (Balami, 2003), plusieurs villes du pays regorgent de petits ou gros élevages, à but lucratif ou non. Abidjan, la capitale économique du pays, présente elle aussi des activités d'élevage (en particulier les bovins de race lagunaire), non négligeable dans la ville et ses environs (CIPEA, 1979; Yapi-Gnaoré et al., 1996). La source d'alimentation de ces bovins est constituée principalement par les ressources fourragères naturelles disponibles (Kouassi et al., 2010) alors que, la prospérité des troupeaux, et parfois même leur survie, est fonction de la quantité, de la qualité et de la continuité de l'affouragement du bétail (Bernard, 2001). La dynamique de développement

\section{MATÉRIEL ET MÉTHODES}

L'étude a été menée dans la zone côtière "Est » du cordon littoral, comprise entre les communes de PortBouët et de Grand-Bassam et est située entre $05^{\circ} 10^{\prime} 54^{\prime \prime}$ de latitude Nord et $03^{\circ} 42^{\prime} 52^{\prime \prime}$ de longitude ouest. Cette zone appartient au secteur littoral du domaine guinéen. C'est une savane côtière qui présente une vaste formation herbeuse avec de rares arbustes groupés en buissons ou forts isolés sur un sol sableux. On y de la ville et la forte urbanisation occasionnent une pression, voire même la disparition des lieux d'approvisionnement et de pâture des animaux (Ali et al., 2003) et aussi, un effet non négligeable sur la paupérisation et la dégradation de la sécurité alimentaire des ménages (Mougeot, 1994 ; ArmarKlemesu, 2000). II en résulte une occupation progressive des espaces anciennement pâturés et la destruction des végétations herbacées et arbustives. Le problème de l'alimentation des bovins liée au développement de la ville constitue donc un frein à la pratique de l'élevage (Kouassi, 2013). Ainsi, la connaissance de la contribution de l'axe Port-Bouët Grand Bassam à l'alimentation des bovins est un élément de base pour attirer l'attention des autorités administratives quant aux conséquences de l'urbanisation non contrôlée sur le développement de l'élevage urbain dans la ville d'Abidjan. C'est dans cet optique que cette étude a été initiée afin d'évaluer le potentiel de production fourragère de la zone pâturée du cordon littoral Port-Bouët - GrandBassam menacée de destruction par les constructions immobilières. Spécifiquement il s'agira de déterminer (1) l'importance pastorale des espèces fourragères présentes, (2) la production fourragère de la zone pâturée.

rencontre des cocoteraies, des maraîchers, des jachères, un fourré littoral, une exploitation d'anacarde. Plusieurs villages de différentes tailles bordent le long de l'axe Port Bouet-Grand Bassam. Aussi, on note la présence de constructions immobilières due à la démographie galopante et à l'urbanisation de la ville d'Abidjan. Les populations vivant dans cette zone, pratiquent de 


\section{Kouassi et al. J. Appl. Biosci. 2014. Potentiel de production fourragère d'une zone pâturée menacée de destruction : cas du cordon littoral Port-Bouët et Grand -Bassam}

nombreuses activités telles que le commerce, l'artisanat, l'élevage et l'agriculture.

Collecte des données : Le dispositif expérimental est constitué de parcelles choisies sur la base des conditions de drainage du sol, de formations végétales en place et du parcours des animaux lors du pâturage. Ainsi fourré littoral, jachère et cocoteraie ont été échantillonnés. La méthode utilisée est celle des relevés phytosociologiques réalisés à l'intérieur de placettes de $25 \mathrm{~m} \times 25 \mathrm{~m}$, obtenues à partir de l'aire minimale. Au total, 110 relevés ont été effectués à partir de 11 transects tracés dans la direction Sud-Nord. Les relevés n'ont pris en compte que les herbacées et les arbustes présents dans les trois écosystèmes précités et aussi sur l'ensemble de la zone d'étude. Ils ont été réalisés selon la méthode sigmatiste classique de Braun-Blanquet (1932). Cette méthode a fait l'objet de nombreuse études réalisées par des auteurs étudiant la végétation intertropicale (Habiyaremye, 1997 ; Ganglo, 1999). Pour chaque relevé, sont notées les informations suivantes : localisation, nature du substrat (boue, sable, vase, etc....), date et numéro de récolte des espèces présentes, indices d'abondance dominance. Les relevés ont permis d'établir la liste de toutes les espèces recensées et de calculer leur contribution spécifique.

Détermination de la Biomasse végétale et la productivité : La biomasse est la masse végétale vivante ou morte sur pied (Daget et Godron, 1995). Selon ces auteurs, la biomasse mesurée à la période de la fructification des herbacées est une bonne expression de la productivité. Pour l'évaluer, la méthode de la récolte intégrale a été utilisée. C'est la méthode la plus directe pour la mesure de la biomasse. Même si elle est destructrice, longue et souvent fastidieuse, elle a l'avantage d'être simple, précise et constitue un outil de terrain particulièrement fiable (Fournier, 1991). La récolte se fait à l'intérieur des placeaux unitaires de $1 \mathrm{~m}^{2}$ (Grouzis, 1988 ; Boudet, 1991). Le nombre de placeaux varie en fonction du niveau de précision recherché, de l'homogénéité du terrain ainsi que des moyens humains et matériels dont on dispose. La récolte de biomasse peut se faire sur des surfaces carrées, rectangulaires ou circulaires (Fournier, 1991). Nous avons opté pour la forme carrée. Ainsi, sur les zones de pâturage, des placeaux repartis au hasard ont été fauchés. L'herbe à l'extérieur du carrée est soigneusement écartée et celle à l'intérieur fauchée. Les carrées avaient un couvert herbacé abondant, homogène et sans signe évident de tonte car les surfaces de l'échantillon n'avaient pas été protégés. La fauche est totale à l'intérieur du carré à une hauteur de $5 \mathrm{~cm}$ du sol. Après la fauche, les opérations suivantes ont été réalisées : le relevé du poids humide de la biomasse totale à l'aide d'un peson de $5 \mathrm{~kg} \pm 50 \mathrm{~g}$; la réalisation d'un tri manuel en séparant les Poaceae fourragères, les Légumineuses fourragères des autres espèces végétales qui ont été considérées comme espèces fourragères. L'herbe ainsi récoltée jusqu'à $5 \mathrm{~cm}$ du sol est pesée à l'état frais sur le site. Après la pesée, nous avons prélevé un échantillon des Poaceae et légumineuses fourragères, et de celui constitué de toutes les autres espèces. Nous les avons pesés et étiquetés, puis les avons placés dans des sachets plastiques. Les échantillons sont séchés à l'étuve à $60^{\circ} \mathrm{C}$ pendant plusieurs jours jusqu'à l'obtention d'un poids sec constant à l'issue de deux ou trois pesées au laboratoire (la seconde fraction étant déduite).

Étude de la valeur pastorale : La valeur pastorale est un indice caractéristique de la valeur d'un pâturage de bovins et d'ovins, à partir d'un relevé en contribution spécifique, par la méthode de relevé selon BraunBlanquet ou par toute autre méthode (Delpech, 1960 ; Daget et Poissonet ,1972). II tient compte de l'abondance relative des espèces, mesurée par leur Contribution Spécifique (CS) et de leur qualité mesurée par l'Indice Spécifique. L'Indice Spécifique (Is) s'obtient soit en considérant la valeur bromatologique d'espèces, ou en s'appuyant sur le classement des espèces par les bergers. En ce qui nous concerne, nous avons utilisé cette deuxième méthode. Aussi une comparaison de notre liste d'espèces à celle relevé dans la littérature a été observée.

\section{Méthode d'analyse des données}

Diversité quantitative: L'approche quantitative concerne la fréquence spécifique et le calcul des contributions spécifiques de présence.

La fréquence spécifique de chaque espèce (FS) s'obtient en faisant la somme des présences de chaque espèce dans chaque relevé ; elle est une valeur absolue;

La contribution spécifique de présence (CSp) correspond à la proportion de chaque espèce présente, exprimée en pourcentage, selon la formule suivante :

$$
C S i=\frac{F S i}{\sum F S i} \times 100
$$

Avec CSi - contribution spécifique et FSi - fréquence spécifique de l'espèce i.

Productivité et capacité de charge de la zone de pâture : La productivité $(P)$ est la production végétale obtenue par la variation de la biomasse $(B)$ dans un intervalle de temps donné ( $t_{1}$ et $\left.t_{2}\right)$.

$P=\Delta B\left(t_{1}\right)-\Delta B\left(t_{2}\right)$

La Capacité de Charge $(C C)$ est un rapport qui permet de déterminer jusqu'à quel niveau un pâturage peut être 


\section{Kouassi et al. J. Appl. Biosci. 2014. Potentiel de production fourragère d'une zone pâturée menacée de destruction : cas du cordon littoral Port-Bouët et Grand -Bassam}

exploité sans risque de voir le pâturage se dégrader, ou le bétail sous alimenté (Breman et Ridder, 1991). Elle peut être exprimée soit en nombre d'unité de bétail tropicale (UBT) par hectare pâturé, soit en nombre d'hectares nécessaires pour nourrir une UBT pendant une période donnée (Beagges, 2003). Elle peut être aussi exprimée en $\mathrm{kg}$ de poids vif à l'ha, ou encore en nombre de jours de pâture d'une UBT. Selon Boudet (1984), le poids des bovins tropicaux oscille entre 200 à $400 \mathrm{~kg}$ ce qui permet de définir l'unité bovin tropical (UBT) comme correspondant a un bovin de $250 \mathrm{~kg}$ à l'entretien. Aussi, selon ce même auteur, on estime à $1 / 3$ de la biomasse primaire, le disponible fourrager réellement consommable par les bovins et à $6,25 \mathrm{~kg}$ la consommation journalière en matière sèche de l'UBT en pâturage sans contrainte. Dans l'estimation de la Capacité de Charge de notre zone d'étude, l'hypothèse que I'UBT (Unité de Bétail Tropical de $250 \mathrm{~kg}$ de poids vif) doit consommer une quantité de $6,25 \mathrm{~kg}$ de matière sèche par jour est adoptée. Ainsi la charge animale pour une période donnée, exprimée en U.B.T./ha est calculée comme suit :

CC $($ UBT $/$ ha $/$ an $)=$ Production $(\mathrm{kg} \mathrm{MS} \mathrm{/} \mathrm{ha)} \times \mathrm{k} / 6,25$ ( $\mathrm{kg} \mathrm{MS/} \mathrm{UBT)} x$ durée d'utilisation (3)

6,25 = consommation journalière d'une UBT (UBT : Unité de bétail Tropical) ; $k=$ coefficient d'utilisation $(k=0,33)$; MS : matière sèche

Valeur pastorale: La Valeur Pastorale (VP) des herbages se détermine à partir d'indice global de qualité et de la contribution spécifique (Csi) du tapis herbacé (Daget et Poissonet, 1971). L'indice tient compte à la fois

\section{RÉSULTATS}

\section{Importance pastorale des espèces rencontrées}

Diversité floristique fourragère : Au total, 190 espèces ont été répertoriées. Elles sont réparties en 152 genres appartenant à 63 familles botaniques. Les quatre familles les mieux représentées constituent $38 \%$ de la flore totale. II s'agit de la famille des Poaceae qui, avec 23 espèces, représente à elle seule, $12,11 \%$ de la flore inventoriée ; les trois autres familles sont les Fabaceae (19 espèces), les Cyperaceae (17 espèces) et les Rubiaceae (15 espèces), renfermant, respectivement, $10 \%, 8,95 \%$ et $7,89 \%$ des espèces de la même flore. L'analyse de la distribution floristique des espèces fourragères montre une dominance de certaines espèces de la zone d'étude. Andropogon canaliculatus Schumach. (Poaceae), espèce à recouvrement moyen de 14,36 p.c. de la surface totale pâturée est la plus rencontrée. Les espèces telles que Eragrostis domingensis (Pers.) Steud. (Poaceae), Digitaria horizontalis Willd. (Poaceae) sont recouvrâtes de la composition spécifique et de l'indice de qualité spécifique (Isi).

L'indice spécifique traduit l'intérêt zootechnique, c'est-àdire la valeur bromatologique de chaque espèce végétale. II a été établi en croisant les données sur l'appétibilité des espèces et celle de la littérature. II est établi sur une échelle de cotation de 0 à 3 (Barral et al., 1983 ; PNUE, 1988), c'est-à-dire sur une échelle de quatre classes $(0,1,2$ et 3$)$ attribué aux espèces végétales selon la grille suivante :

- les plantes de bonne valeur pastorale (BVP) sont celles dont l'/si st égal à 3 ;

- les plantes de valeur pastorale moyenne (MVP) sont celles dont l'/si est égal à 2 ;

- les plantes de valeur pastorale faible (FVP) sont celles dont l'/si est égal à 1 ;

- les plantes sans valeur pastorale (SVP) sont celles dont l'Isi est égal à 0 .

Ces différents groupes d'espèces (BVP, MVP, FVP et SVP) ou classes d'indices constituent dans la suite du texte les différentes catégories d'espèces fourragères.

La valeur pastorale a été calculée en multipliant les contributions des espèces (Csi) par les indices de qualité correspondants $(I S I)$; les valeurs pastorales relatives $(\mathrm{VPr})$ des espèces ainsi obtenues sont additionnées puis divisées par le nombre de classes significatives d'indices $(I s i \neq 0)$ et exprimées en pourcentage (Daget et Poissonet, 1971):

$V P=\frac{1}{3 \sum C S i \times I S i}$

respectivement à 6,54 p.c. et 2,35 p.c. de la surface totale de la flore globale. Aussi, les Poaceae fourragères avec 11,04 p.c. d'espèces fourragères sont les plus nombreuses. Les Légumineuses fourragères représentent 7,36 p.c. et les autres fourrages constituent en moyenne 9 p.c. de la flore totale.

Contribution spécifique relative des principales espèces fourragères : La contribution spécifique des différentes espèces selon le type de fourrage est présentée par la figure 1. Les Poaceae fourragères sont plus représentatives dans les cocoteraies et les jachères avec respectivement des contributions de 20,16 p.c. et 15,64 p.c. Les Légumineuses fourragères sont plus importantes dans les jachères que dans les autres écosystèmes. Les autres espèces fourragères quant à elles sont très représentées dans les cocoteraies et les jachères. Aussi, la contribution spécifique des plantes 
herbacées fourragères de la zone de pâture est de $57,10 \quad$ p.c.

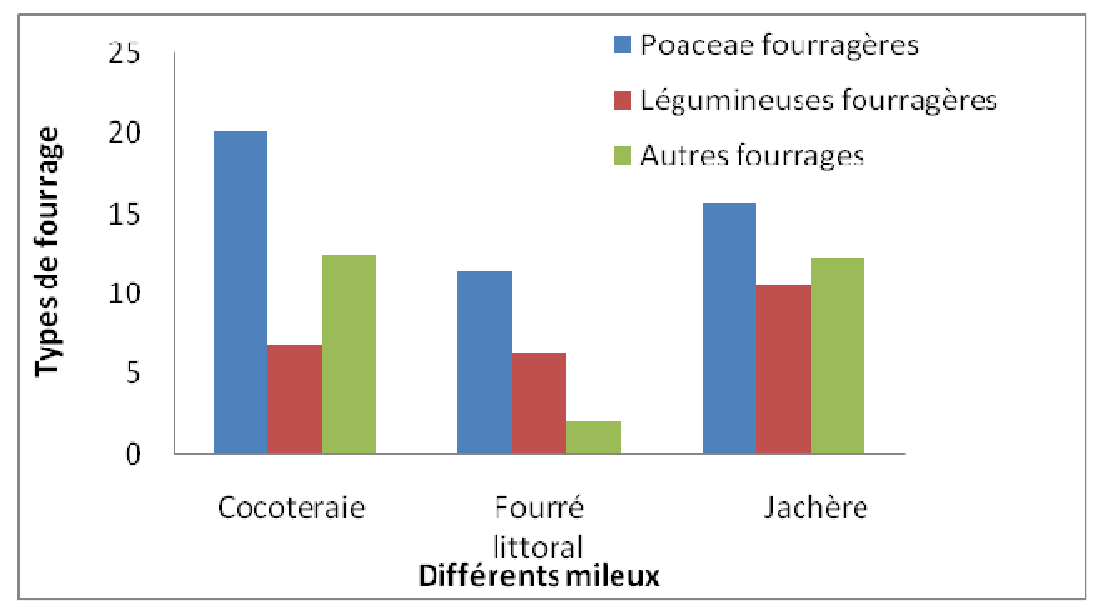

Figure 1 : Contribution spécifique relative des principaux types de fourrages rencontrés.

Production fourragère de la zone Port-Bouët à Grand Bassam

Valeur pastorale de la zone pâturée (VP): La classification des espèces en fonction de l'indice de qualité fourragère montre que les espèces végétales à valeur fourragère moyenne ont été les plus recensées avec un pourcentage de 49,42 suivit des espèces fourragères à faible valeur et enfin celles à bonne valeur fourragère. Concernant la valeur pastorale de la zone de pâture, elle est relativement faible avec un pourcentage de 40,91 (Tableau 1).

Tableau $1:$ Indice de qualité fourragère (p.c.) et valeur pastorale brute des écosystèmes étudiés.

\begin{tabular}{lllll}
\hline Indice de qualité & Zone d'étude & Cocoteraie & Fourré littoral & Jachère \\
\hline Faible valeur & 27,05 & 28,38 & 37,15 & 22,22 \\
Moyenne valeur & 49,42 & 55,40 & 51,42 & 40,00 \\
Bonne Valeur & 23,53 & 16,21 & 11,43 & 37,78 \\
VP totale & 40,91 & 40,73 & 35,7 & 55,7 \\
\hline
\end{tabular}

Productivité et capacité de charge des zones de pâture: Les rendements bruts de biomasse primaire obtenus sur les sites pâturés dans la période de Mars à juin 2007 montrent des productions moyennes, relativement faibles au niveau des jachères et des cocoteraies. Elles sont respectivement de $554,77 \mathrm{Kg}$ MS./ha et 438,54 Kg MS./ha. Concernant le fourré littoral, elle présente une production moyenne de $1236,9 \mathrm{Kg}$ MS./ha qui est le double de celles de chacune des deux autres écosystèmes pâturés. La production potentielle, qui représente la biomasse du couvert herbacé pendant la période active du pâturage à l'abri des herbivores est respectivement de $408,12 \mathrm{Kg} \mathrm{MS} / \mathrm{ha} ; 183,07 \mathrm{Kg} \mathrm{MS} / \mathrm{ha}$; $144,71 \mathrm{Kg} \mathrm{MS} / \mathrm{ha}$ pour le fourré littoral, la jachère et la cocoteraie. Ces productions potentielles correspondent à des capacités de charge moyenne de 1,87 ha/U.B.T. de pâturage pour le fourré littoral, 4,16 ha/U.B.T. pour la jachère, 5,27 ha/U.B.T pour la cocoteraie et 1 ha de pâturage par U.B.T. pour la zone totale (Tableau 2).

Tableau 2: Productivité fourragère et capacité de charge des différentes zones pâturées.

\begin{tabular}{c|c|c|c|c|c|c}
\hline Végétations & $\begin{array}{c}\text { Rendement } \\
\text { (Kg.F./ha) }\end{array}$ & $\begin{array}{c}\text { Rendement } \\
\text { (Kg.S./ha) }\end{array}$ & $\begin{array}{c}\text { Production potentielle } \\
\text { (Kg.S./ha) }\end{array}$ & $\begin{array}{c}\text { Nombre de } \\
\text { jours pâturés }\end{array}$ & $\begin{array}{c}\text { CC } \\
\text { U.B.T./ha }\end{array}$ & $\begin{array}{c}\text { CC } \\
\text { ha/U.B.T }\end{array}$ \\
\hline Fourré littoral & 3265,6 & 1236,9 & 408,12 & 65 & 0,53 & 1,87 \\
Jachère & 1586,19 & 554,77 & 183,07 & 29 & 0,24 & 4,16 \\
Cocoteraie & 1454 & 438,54 & 144,71 & 23 & 0,19 & 5,27 \\
Zone totale & 6305,79 & 2230,21 & 735,96 & 118 & 0,96 & 1,03 \\
\hline
\end{tabular}




\section{Kouassi et al. J. Appl. Biosci. 2014. Potentiel de production fourragère d'une zone pâturée menacée de destruction : cas du cordon littoral Port-Bouët et Grand -Bassam}

\section{DISCUSSION}

L'état d'un pâturage dépend avant tout de la proportion des Graminées, les espèces les plus productives et les plus consommées par le bétail (Fournier, 1991 ; Cesar, 1992). Dans notre zone d'étude les Poaceae représentent la plus grande proportion de toutes les espèces recensées. La forte proportion des Poaceae peut s'expliquer par le fait qu'elles possèdent une très grande possibilité de tallage et de repousse après broutage, lorsque les conditions du milieu sont favorables.Les contributions spécifiques des herbacées diverses consommées sont supérieures à 50 p.c.. Cela signifie que la proportion de plantes herbacées contribue à l'alimentation des bovins présents sur la zone d'étude. Des valeurs similaires ont été trouvées par Hoffmann (1985) qui a analysé la flore des jachères pâturées en pays Lobi au Nord-Est de la Côte d'Ivoire. L'auteur évalue la proportion des herbacées diverses à 50,5 p.c., alors qu'elle est comprise entre 3 et 14 p.c. dans les jachères voisines non pâturées. Aussi, des travaux en zone sahélienne du Niger montrent qu'en cas de pâturage important dans des écosystèmes pâturés en saison de pluie, la végétation est dominée par une espèce ou par un petit nombre d'espèces qui représentent en moyenne 50 p.c. de la contribution spécifique des espèces recensées (Banon et Achard, 1998). La présence d'espèces indicatrices de mauvaise gestion pastorale, voire de surpâturage en agrostologie, n'a pas été recensée dans les écosystèmes pâturés. II s'agit d'espèces classées dans la catégorie des refus, comme Elionurus pobeguinii Stapf. J.P. Lebrun (Poaceae), Panicum phragmitoides Stapf. (Poaceae) et certaines espèces du genre Spermacoce qui se multiplient au détriment des espèces de bonnes valeurs fourragères. Ces espèces sont considérées comme indicatrices de surpâturage (Cesar, 1992). Les indices spécifiques sont constants dans le temps et dans l'espace, ce qui diffère des propriétés nutritives d'une espèce, qui varient selon les saisons et les écotypes (Akpo et Grouzis, 2000). La contribution spécifique tient compte aussi bien des espèces fourragères que des espèces non fourragères. L'espèce Andropogon canaliculatus a la contribution spécifique la plus élevée avec plus de 5 p.c. sur la totalité de la zone d'étude. Certaines espèces telles que Perotis indica, Eriosema glomeratum, Ageratum conyzoides présentent des contributions spécifiques supérieures à 3 p.c.. Cela signifie que ces espèces sont les plus rencontrées. En plus des espèces fourragères, on note aussi que certaines espèces herbacées non fourragères telles que Diodia rubricosa Hiern. (Rubiaceae) et bien d'autres présentent une contribution spécifique supérieure à 2 p.c.. La présence de ces espèces aurait ainsi contribué au faible taux du potentiel fourrager dans la zone d'étude. Selon Garde et Senn (1991), la présence d'espèces non fourragères dans un parcours entraîne une baisse de la valeur pastorale. Des auteurs se sont demandé comment cette baisse se justifiait alors que les espèces non fourragères n'entravaient pas la consommation des espèces fourragères. Toujours Selon Garde et Senn (1991), la composante animale n'est pas prise en compte dans le calcul de la valeur pastorale. En effet, le type d'animal et les modalités de pâturage ne sont pas spécialement intégrés dans le mode d'évaluation. II en est de même pour le comportement alimentaire, car les animaux choisissent ce qu'ils mangent et la végétation ingérée est parfois différente de la végétation sur pied (Garde et Senn 1991).La mesure de la production potentielle en fin de période et la détermination de la période active sont la base de la détermination des capacités de charge des pâturages naturels à défaut d'expérimentation zootechnique. La production potentielle obtenue dans la zone pâturée est de $735,96 \mathrm{Kg}$ de MS/ha en 4 mois d'exploitation. Elle a permis d'obtenir une capacité de charge de 1,03 ha de pâturage par U.B.T., c'est-à-dire il faut en moyenne un hectare pour chaque U.B.T. qui pâture la zone. La capacité de charge en UBT/ha, qui correspond au nombre de têtes de bétail, qu'un parcours peut accueillir et nourrir sans se détériorer est de 0,96 UBT/ha. Cela signifie qu'en moyenne un hectare de la zone d'étude peut nourrit correctement un bovin de $250 \mathrm{~kg}$ de poids vif. Ce résultat est proche de celui de Akpo et al. (2002) qui a obtenu une capacité de charge comprise entre 0,91 et $0,93 \mathrm{UBT} / \mathrm{ha}$ dans plusieurs zones de parcours. Toutefois il est différent de celui de Boudet (1991), qui selon lui, la production potentielle suivant le type de sol dans les pâturages guinéens varie de 2.5 à 3 tonnes de MS/ha à 13 tonnes de MS/ha, soit une capacité de charge de 145 jours UBT à 690 jours UBT de pâture. Ainsi pour maintenir un niveau d'exploitation durable de la zone étudiée, il serait important de respecter la capacité de charge obtenue et de mettre en place un système permettant de réglementation de la pâture des bovins sur le site d'étude. 


\section{Kouassi et al. J. Appl. Biosci. 2014. Potentiel de production fourragère d'une zone pâturée menacée de destruction : cas du cordon littoral Port-Bouët et Grand -Bassam}

\section{CONCLUSION}

Au total 190 espèces ont été répertoriées. Les Poaceae, Fabaceae et Cyperaceae sont les familles les mieux représentées. Parmi les espèces recensées, les plus appétées sont Andropogon canaliculatus Schumach. (Poaceae) Stylosanthes fruticosa (Retz.) Alston (Fabaceae) et Eriosema glomeratum (Guill. \& Perr.) Hook.f. (Fabaceae). Toutes ces espèces citées contribuent de manière significative à la production de biomasse. La productivité fourragère représente en moyenne un hectare de pâturage pour 1 U.B.T. Au regard de tout cela, nous pouvons dire que la zone entre Port-

\section{RÉFÉRENCES BIBLIOGRAPHIQUES}

Akpo LE. et Grouzis M, 2000. Valeur pastorale des herbages en région soudanienne : le cas des parcours sahéliens du Nord-Sénégal. Tropicultura, 18: 1-8.

Akpo L E, Masse D, Grouzis M, 2002. Durée de jachère et valeur pastorale de la végétation herbacée en zone soudanienne au Sénégal. Revue d'élevage et de médecine vétérinaire des pays tropicaux, 55(4) : 275-283.

Ali L, Van den Bossche P, Thys E, 2003. Enjeux et contraintes de l'élevage urbain et périurbain des petits ruminants à Maradi au Niger : quel avenir ? Revue Élev.Méd.vét. Pays trop, 56 (1-2): 7382

Armar-Klemesu M, 2000. Urban agriculture and food security, nutrition and health. In: Bakker N., Dubbeling M., Gündel S., Sabel-Koshella U., de Zeeuw H., Eds, Growing cities, growing food: urban

agriculture on the policy agenda, Proc. Workshop in La Havana, Cuba, 11-15 October 1999. Feldafing, Germany, DSE-ZEL, pp. 99-117.

Balami $\mathrm{D} \mathrm{H}, 2003$. Le rôle de la filière bétail dans la compétitivité des pays ouest-Africains. In : Club du sahel, l'Afrique de l'Ouest et la mondialisation : Les Facteurs de la compétitivité, pp. 235-257.

Banon M et Achard F, 1998. Place de la jachère dans les systèmes d'élevage et Comportement alimentaire du bétail sur un terroir du sud ouest du Niger : Tiko. In : Jachère et systèmes agraires. Actes de l'atelier de Niamey, Niger, 30 septembre au 2 octobre 1998, pp. 99-110.

Barra I H, Bénéfice E, Boudet G, Dénis JP, 1983. Systèmes de production d'élevage au Sénégal dans la région du Ferlo : synthèse de fin
Bouët et Grand-Bassam joue un rôle important dans l'alimentation des bovins. Cette zone serait propice à abriter de l'élevage de grande taille à condition de réorganiser et d'améliorer le secteur de l'alimentation des bovins. Aussi, il serait judicieux d'attirer l'attention des autorités quant à la destruction de la zone au profit des constructions immobilières. La présence d'un élevage de grande taille constituerait une importante source de revenus et jouerait un grand rôle dans l'approvisionnement en viande des abattoirs de la ville d'Abidjan.

d'études d'une équipe de recherches pluridisciplinaires. ACC - GRIZA (LAT): 172 p.

Beagges S, 2003. Caractéristiques sociologiques et socio pastorales des villages de la zone du projet moyen Bani. Bamako, Mali, $32 \mathrm{p}$.

Bernard T, 2001. Programme Écosystèmes Naturels et Pastoraux Campus International de Baillarguet, TA 30/F, 34398 - Montpellier Cedex 05, France CIRAD-EMVT Source : Élevage et gestion de parcours au Sahel, implications pour le développement

Braun-Blanquet J, 1932. Plant sociology. The study of plant communities (English translation of "pflazensoziologie" by G. D. Fuller and H.S. Conard) Université de Chicago (USA), $439 \mathrm{p}$.

Breman $\mathrm{H}$ et Ridder N, 1991. Manuel sur les pâturages des pays sahéliens. ACCT-CTAKARTHAL, A, $47 \mathrm{p}$.

Boudet G, 1984. Manuel sur les pâturages tropicaux et les cultures fourragères. 4 éd. Ministère de la Coopération de la France, Paris Manuels et Précis d'Élevage 4: 1-254.

Boudet G, 1991. Manuel sur les pâturages tropicaux et les cultures fourragères. Manuel et précis d'élevage (1ère édition 1974). IEMVT/Ministère de la coopération de la France, Paris, $266 \mathrm{p}$.

Cesar J, 1992. Étude de la production biologique des savanes de la Côte d'Ivoire et son utilisation par l'homme. Biomasse, valeur pastorale et production fourragère. Doctorat d'État, Université Pierre et Marie Curie, Paris, France, $672 p$.

C.I.P.E.A., 1979. Centre international pour l'élevage en Afrique. Monographie 2. Le bétail trypanotolérant d'Afrique Occidentale et centrale. Tome 1, Situation Générale : AddisAbeba, $155 \mathrm{p}$. 


\section{Kouassi et al. J. Appl. Biosci. 2014. Potentiel de production fourragère d'une zone pâturée menacée de destruction : cas du cordon littoral Port-Bouët et Grand -Bassam}

Daget P. et Poissonet J, 1971. Une méthode d'analyse phytologique des prairies. Critères d'application. Annales Agronomiques, 22: 5-41.

Daget $P$ et Poissonet J, 1972. Un procédé d'estimation de la valeur pastorale des pâturages. Fourrages, 49: 31-39.

Daget P et Godron M, 1995. Pastoralisme : troupeaux, espaces et sociétés. Hatier/Aupelf, Paris, France, $510 \mathrm{p}$.

Delpech R, 1960. Critères et jugement de la valeur agronomique des prairies. Ann Agro., (212) : 541.

Fournier A, 1991. Phénologie, croissance et production végétales dans quelques savanes d'Afrique de I'Ouest. ORSTOM, Paris: 1-312

Ganglo C. J., 1999. Poster presented at the Proceedings of the XVIth International Botanical Congress. August 1-7, 1999, Saint Louis, Missouri, USA. $531 \mathrm{p}$.

Garde L et Senn O, 1991 Valeur pastorale et parcours méditerranéens. In : Gaston et coll. éds, actes 4è Congrès international des terres de parcours, Montpellier, France, avril 1991, pp. 458-461.

Grouzis M, 1988. Structure, productivité et dynamique des systèmes écologiques sahéliens (Mare d'Oursi, Burkina Faso). ORSTOM, Paris, 139 p.

Habiyaremye F.X., 1997. Étude phytosociologique de la dorsale orientale du lac Kivu. Musée Royal de l'Afrique Centrale, Tervuren, Belgique, Annales Sciences Economiques, 24 : 276 p.

Hoffmann O., 1985. Pratiques pastorales et dynamiques du couvert végétal en pays Lobi (nord-est de la Côte d' Ivoire). ORSTOM, Paris, France, 355 p.

Kouassi AF, 2013. Étude Agrostologique et Socioéconomique des exploitations fourragères dans les zones urbaines et périurbaines da la ville d'Abidjan (Côte d'Ivoire).Thèse Unique, UFR Biosciences, Université Félix Houphouët Boigny Abidjan, $173 p$.

Kouassi AF, Majorein V, Ipou IJ, Adou Yao CY, Kamanzi $\mathrm{K}, 2010$. Alimentation des ovins des marchés de vente de bétail dans la ville d'Abidjan, Côte d'Ivoire. Agronomie Africaine, 22 (1): 1-95 ISSN $n^{\circ} 1015-2288$.

Mougeot LJA, 2000. Urban agriculture: definition, presence, potential and risks. In :Bakker N. et al., Growing cities, growing food: urban agriculture on the policy agenda, a reader on urban agriculture. Feldafing, Allemagne, DesEtc, $18 p$.
Mougeot LJA, 1994. Urban food production : evolution, official support and significance, with special reference to Africa. Ottawa, Canada, IDRC.

Moustier PA et Mbaye H, 1999.Agriculture Periurbaine en Afrique Subsaharienne. Workshop 20-24 April 1998, Montpellier, France, Cirad/Coraf, pp. 7-16.

PNUE, 1988. Introduction au projet Écosystèmes pastoraux sahéliens : rapport général. Gems, Série : Sahel. Rome, Italie, PNUE/ONU-AA, 145 p.

Yapi-Gnaoré C V, Oya B, Ouattara Z, 1996. Revue de la situation des races d'animaux domestiques de Côte d'Ivoire Animal Genetic Ressources Information, 19: 99-118.

Waters-Bayers A, 2000. Living with livestock in town. UrbanAgric. Mag., 1: 16-20. 